\title{
Combination immunohistochemistry for SMAD4 and Runt- related transcription factor 3 may identify a favorable prognostic subgroup of pancreatic ductal adenocarcinomas
}

\author{
Yangkyu Lee ${ }^{1}$, Hyejung Lee ${ }^{2}$, Hyunjin Park², Jin-Won Kim³, Jin-Hyeok Hwang ${ }^{3}$, \\ Jaihwan Kim ${ }^{3}$, Yoo-Seok Yoon ${ }^{4}$, Ho-Seong Han ${ }^{4}$ and Haeryoung Kim² \\ ${ }^{1}$ Department of Pathology, Seoul National University Bundang Hospital, Seoul National University College of Medicine, \\ Seongnam, Republic of Korea \\ ${ }^{2}$ Department of Pathology, Seoul National University Hospital, Seoul National University College of Medicine, Seoul, Republic \\ of Korea \\ ${ }^{3}$ Department of Internal Medicine, Seoul National University Bundang Hospital, Seoul National University College of Medicine, \\ Seongnam, Republic of Korea \\ ${ }^{4}$ Department of Surgery, Seoul National University Bundang Hospital, Seoul National University College of Medicine, \\ Seongnam, Republic of Korea \\ Correspondence to: Haeryoung Kim, email: haeryoung.kim@snu.ac.kr
}

Keywords: pancreatic ductal adenocarcinoma, SMAD4, RUNX3, immunohistochemistry, prognosis

Received: June 05, 2017

Accepted: August 23, 2017

Published: September 11, 2017

Copyright: Lee et al. This is an open-access article distributed under the terms of the Creative Commons Attribution License 3.0 (CC BY 3.0), which permits unrestricted use, distribution, and reproduction in any medium, provided the original author and source are credited.

\section{ABSTRACT}

Purposes: SMAD4/DPC4 mutations have been associated with aggressive behavior in pancreatic ductal adenocarcinomas (PDAC), and it has recently been suggested that RUNX3 expression combined with SMAD4 status may predict the metastatic potential of PDACs. We evaluated the prognostic utility of SMAD4/RUNX3 status in human PDACs by immunohistochemistry.

Materials and Methods: Immunohistochemical stains were performed for SMAD4 and RUNX3 on 210 surgically resected PDACs, and the results were correlated with the clinicopathological features.

Results: Loss of SMAD4 expression was associated with poor overall survival (OS) $(p=0.015)$ and progression-free survival (PFS) $(p=0.044)$. Nuclear RUNX3 expression was associated with decreased OS $(p=0.010)$ and PFS $(p=0.009)$, and more frequent in poorly differentiated PDACs $(p=0.037)$. On combining RUNX3/ SMAD4 status, RUNX3-/SMAD4+ PDACs demonstrated longer OS ( $p=0.008$, median time; RUNX3-/SMAD4+ 34 months, others 17 months) and PFS ( $p=0.009$, median time; RUNX3-/SMAD4+ 29 months, others 8 months) compared to RUNX3+/SMAD4+ and SMAD4- groups; RUNX3-/SMAD4+ was a significant independent predictive factor for both OS $[p=0.025$, HR $1.842(95 \%$ CI 1.079-3.143)] and PFS $[p=0.020$, HR 1.850 (95\% CI 1.100-3.113)].

Conclusions: SMAD4-positivity with RUNX3-negativity was a significant independent predictive factor for favorable OS and PFS in PDAC. This is the first and large clinicopathological study of RUNX3/SMAD4 expression status in human PDAC. Combination immunohistochemistry for SMAD4 and RUNX3 may help identify a favorable prognostic subgroup of PDAC.

\section{INTRODUCTION}

Pancreas cancer is the fourth most common cause of cancer-related deaths in industrialized countries, with an overall 5 -year survival rate of about $8 \%$ [1]. The majority of patients are diagnosed at an advanced stage with distant metastasis or invasion of adjacent organs, and less than $20 \%$ of patients are amenable to curative resection [1].

The common genetic alterations in pancreatic carcinogenesis include activating mutations in $K R A S$, telomere shortening, and inactivation of $C D K 2 N A / p 16$, TP53 and SMAD4 genes [2, 3]. However, the molecular 
landscape of pancreatic ductal adenocarcinomas (PDACs) is extremely vast. For example, recent large-scale genomic analyses on PDACs have identified various subtypes of these cancers highlighting their heterogeneity, e.g. the "Collisson class" (classical, quasimesenchymal and exocrine-like subtypes), "Moffitt tumor class" (basallike versus classical), "Moffitt stromal class" (activated stroma, normal stroma) and the subtypes recently defined by Bailey et al. (squamous, pancreatic progenitor, immunogenic and aberrantly differentiated endocrine exocrine (ADEX) subtypes) [4-6]. Thus, targeting the specific molecular subtypes would ideally help improve the outcomes of PDAC patients, in the way that selected patients with breast and lung cancers have greatly benefitted from targeted therapy over the past decades.

While such high-throughput genomic analyses have yielded promising results, it is also important that inexpensive and easily applicable tests (e.g. immunohistochemical assays) are developed for predicting therapeutic response and prognosis. In this regard, Runtrelated transcription factor 3 (RUNX3) is an interesting candidate biomarker for PDAC, as it has recently been demonstrated to play a role as a "metastatic switch" in PDACs in genetically engineered mouse models: RUNX3 was suggested to be an important determinant of the ability of PDAC cells to metastasize to distant sites or to proliferate locally [7, 8]. Moreover, RUNX3 expression was shown to be influenced by SMAD4 status in the same study: heterozygous deletion of SMAD4 (+/-) attenuated RUNX3 levels compared to intact SMAD4 (+/+) and homozygously-deleted SMAD4 (-/-) [8].

If the combination of RUNX3 and SMAD4 status could predict the behavior of PDAC, analyzing RUNX3 and SMAD4 protein expression status by immunohistochemistry in resected or biopsied PDAC tissues would greatly benefit pancreatic cancer patients by helping to guide treatment plans. For example, PDACs with increased metastatic potential could benefit from systemic chemotherapy, while the more locally proliferative PDACs would be candidates for more aggressive local treatment, such as surgery or radiotherapy. Therefore, in this study, we performed an immunohistochemical analysis of RUNX3 and SMAD4 expression in our cohort of 210 surgically resected PDACs, and compared the clinicopathological features of PDACs according to RUNX3/SMAD4 expression status.

\section{RESULTS}

\section{Clinicopathological characteristics}

The clinicopathological characteristics of 210 cases are summarized in Table 1. $126(60 \%)$ out of 210 patients were male, and the median age at initial operation was 65 years (range: 29 89 years). Most patients underwent operation for initial treatment. Only 7 patients received neoadjuvant chemotherapy before resection. The majority of PDACs were moderately differentiated (159/210, $75.7 \%$ ) and were pT3 by the American Joint Committee on Cancer (AJCC) 7th edition (201/210, 95.7\%). When the recently published size-based AJCC 8 th edition was applied, 12.4\% (26/210) were pT1 and 58.1\% (122/210) were pT2, while $28.5 \%$ and $1.0 \%$ were pT3 and pT4, respectively, at the time of surgery. 130/210 (61.9\%) cases had lymph node metastasis. On follow up, 86/210 (41.0\%) and 121/210 (57.6\%) developed local recurrence and distant metastasis, respectively, and 174/210 (82.9\%) patients were deceased at last follow up.

\section{RUNX3 and SMAD4 expression in pancreatic ductal adenocarcinomas}

The immunohistochemical stain results are demonstrated in Figure 1. Loss of SMAD4 expression ("SMAD4-") was observed in 145 (72.5\%) cases, while the remaining $55(27.5 \%)$ cases had intact SMAD4 ("SMAD4+"). RUNX3 expression ("RUNX3+") was identified in 121 cases $(59.0 \%)$ compared with remaining 84 cases showing no RUNX3 expression ("RUNX3-"). On combining the SMAD4 and RUNX3 status, 25 (11.9\%) PDACs demonstrated intact SMAD4 with no RUNX3 expression (RUNX3-/SMAD4+).

When the clinicopathological features were compared according to SMAD4 and RUNX3 expression status (Table 2), we found SMAD4 loss was associated with increased tumor size $(p=0.030)$, higher $\mathrm{pN}$ stage (AJCC 8th ed.) $(p=0.035)$, poor histological differentiation $(p=0.051)$ and higher $\mathrm{pT}$ stage (AJCC 8th ed.) ( $p=0.054)$, although the latter two parameters were marginally significant. When the disease recurrence patterns were compared, PDACs with SMAD4 loss tended to result more frequently in distant metastasis compared to SMAD4-intact PDACs $(p=0.058)$, while there was no difference in the frequency of local recurrence according to SMAD4 expression status. RUNX3 expression was associated with tumor size $(p=0.039)$ and poor histological differentiation $(p=0.033)$.

On combining the expression status of the two markers, PDACs with RUNX3-/SMAD4+ profiles showed less frequent distant metastasis $(p=0.017)$ and tended to be smaller in size $(p=0.092)$ compared to the other PDACs which showed SMAD4 loss and/or RUNX3 expression (Table 3 ).

\section{Survival analysis}

The univariate survival analysis results are summarized in Table 4 and Figure 2. Clinicopathological parameters significantly associated with poor overall survival included large tumor size $(p=0.032)$, presence of lymphatic $(p=0.005)$, venous $(p<0.001)$ or perineural invasion $(p=0.007)$, high pT stage ( 7 th ed.) $(p=0.041)$ 
Table 1: Patient and tumor characteristics $(n=210)$

\begin{tabular}{|c|c|}
\hline \multicolumn{2}{|l|}{ Sex } \\
\hline Male & $126(60.0 \%)$ \\
\hline Female & $84(40.0 \%)$ \\
\hline Age at operation, median (range, years) & $65(29-89)$ \\
\hline Initial CEA, median (ng/ml) & $3.0(0.01-50.6)$ \\
\hline Initial CA19-9, median (IU/ml) & $121.2(0.01-10000)$ \\
\hline \multicolumn{2}{|l|}{ Tumor size } \\
\hline$\leq 2 \mathrm{~cm}$ & $26(12.4 \%)$ \\
\hline$>2 \mathrm{~cm}$ and $\leq 4 \mathrm{~cm}$ & $123(58.6 \%)$ \\
\hline$>4 \mathrm{~cm}$ & $61(29.0 \%)$ \\
\hline \multicolumn{2}{|l|}{ Preoperative treatment } \\
\hline None & $203(96.7 \%)$ \\
\hline Neoadjuvant chemotherapy & $7(3.3 \%)$ \\
\hline \multicolumn{2}{|l|}{ Histologic grade } \\
\hline Well differentiated & $23(11.0 \%)$ \\
\hline Moderately differentiated & $159(75.7 \%)$ \\
\hline Poorly differentiated & $23(11.0 \%)$ \\
\hline Undifferentiated & $5(2.3 \%)$ \\
\hline \multicolumn{2}{|l|}{ pT stage (AJCC 7th Ed.) } \\
\hline pT1 & $2(1.0 \%)$ \\
\hline pT2 & $5(2.3 \%)$ \\
\hline pT3 & $201(95.7 \%)$ \\
\hline pT4 & $2(1.0 \%)$ \\
\hline \multicolumn{2}{|l|}{ pT stage (AJCC 8th Ed.) } \\
\hline $\mathrm{pT} 1(\leq 2 \mathrm{~cm})$ & $26(12.4 \%)$ \\
\hline $\mathrm{pT} 1 \mathrm{a}(\leq 0.5 \mathrm{~cm})$ & 0 \\
\hline pT1b $(>0.5 \mathrm{~cm}$ and $<1 \mathrm{~cm})$ & 0 \\
\hline $\mathrm{pT} 1 \mathrm{c}(\geq 1 \mathrm{~cm}$ and $\leq 2 \mathrm{~cm})$ & 26 \\
\hline $\mathrm{pT} 2(>2 \mathrm{~cm}$ and $\leq 4 \mathrm{~cm})$ & $122(58.1 \%)$ \\
\hline pT3 $(>4 \mathrm{~cm})$ & $60(28.5 \%)$ \\
\hline pT4 & $2(1.0 \%)$ \\
\hline \multicolumn{2}{|l|}{ pN stage (AJCC 7th Ed.) } \\
\hline pNo & $80(38.1 \%)$ \\
\hline pN1 & $130(61.9 \%)$ \\
\hline \multicolumn{2}{|l|}{ pN stage (AJCC 8th Ed.) } \\
\hline pN0 (no metastasis) & $80(38.1 \%)$ \\
\hline pN1 (1 3) & $97(46.2 \%)$ \\
\hline $\mathrm{pN} 2(\geq 4)$ & $33(15.7 \%)$ \\
\hline \multicolumn{2}{|l|}{ Local recurrence } \\
\hline Absent & $124(59.0 \%)$ \\
\hline Present & $86(41.0 \%)$ \\
\hline \multicolumn{2}{|l|}{ Distant metastasis } \\
\hline Absent & $89(42.4 \%)$ \\
\hline Present & $121(57.6 \%)$ \\
\hline \multicolumn{2}{|l|}{ Status at last follow up } \\
\hline Alive & $36(17.1 \%)$ \\
\hline Deceased & $174(82.9 \%)$ \\
\hline
\end{tabular}


and high pN stage (7th ed. $p<0.001,8$ th ed. $p=0.001$ ). Similar findings were seen for progression-free survival: larger tumor size $(p=0.013)$, presence of lymphatic $(p=0.003)$, venous $(p=0.003)$ or perineural invasion $(p=0.007)$, high pT stage $(7$ th ed. $p=0.014,8$ th ed. $p=0.018)$, and high $\mathrm{pN}$ stage ( 7 th ed. $p=0.003,8$ th ed. $p=0.011)$ were significantly associated with reduced progression-free survival.

When the survival was analyzed according to the immunohistochemical stain results, loss of SMAD4 expression was significantly associated with poor overall survival (median survival 16 months versus 25 months, $p=0.015$ ) and progression-free survival (median survival 8 months versus 11 months, $p=0.044$ ) compared to PDACs with intact SMAD4 expression. RUNX3 expression was significantly associated with decreased overall survival (median survival 16 months versus 23 months, $p=0.010$ ) and progression-free survival (median survival 8 months versus 12 months, $p=0.009$ ) compared to RUNX3negative PDACs. When the RUNX3/SMAD4 status was combined, RUNX3-/SMAD4+ tumors were associated with remarkably longer overall survival (median 34 months versus 17 months, $p=0.008$ ) and progressionfree survivals (median 29 months versus 8 months, $p=$ $0.009)$ compared to other groups. Interestingly, among the PDACs with no RUNX3 expression, those with SMAD4 loss demonstrated similarly decreased overall and progression-free survivals as RUNX3-expressing PDACs.

After adjusting for sex and age, RUNX3-/SMAD4+ status remained a significant independent predictive factor for favorable overall $[p=0.025, \mathrm{HR} 1.842$ (95\% CI $1.079-3.143)]$ and progression-free survival $[p=0.020$, HR 1.850 (95\% CI 1.100-3.113)] on multivariate analysis, in addition to tumor size (Table 5).

\section{Comparison of immunohistochemical status between primary and metastatic lesions}

Of the 210 patients studied, $121 \quad(57.6 \%)$ subsequently developed distant metastases. Biopsies or resected tissues from metastatic sites were available for $14 / 121$ patients and the whole tissue sections of the 14 metastatic tumors were subjected to SMAD4 and RUNX3 immunohistochemistry. The liver was the most common site of distant metastasis $(n=9)$ (Table 6).

Identical RUNX3/SMAD4 profiles were seen in the primary and metastatic tumors of 5/14 (35.7\%) cases. While $11 / 14(78.6 \%)$ of the cases showed concordant SMAD4 expression status between the matched primary and metastatic tumors, only 6/14 (42.9\%) cases showed concordant RUNX3 expression status. Interestingly, out of the 14 matched cases, the RUNX3-/SMAD4+ profile was seen in none of the primary PDACs and also in none of their subsequent metastatic tumors. In addition, only $1 / 14(7.1 \%)$ of the metastatic tumors had intact SMAD4 expression, and all $8(100 \%)$ cases with discordant RUNX3 status were RUNX3+ in the primary tumor and RUNX3-negative in the matched metastatic tumors.
A



D

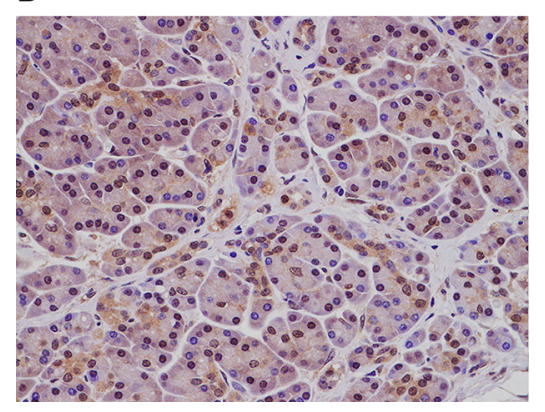

B

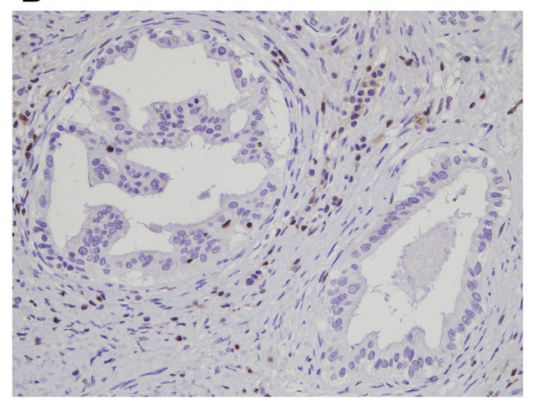

E

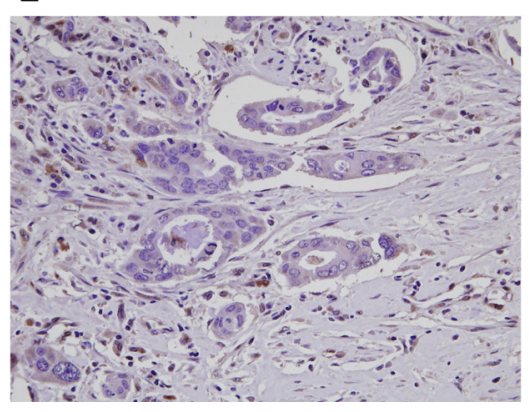

C

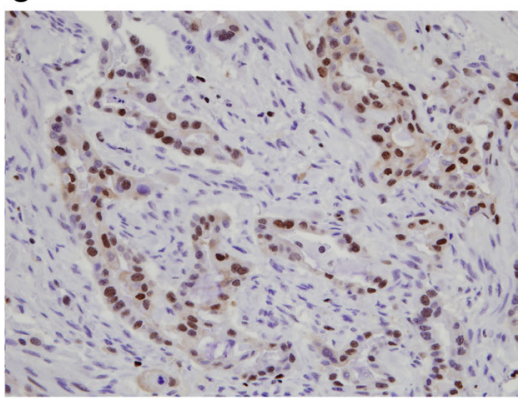

$\mathbf{F}$

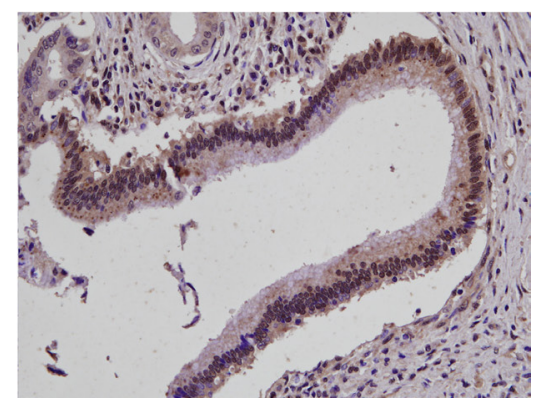

Figure 1: Immunohistochemical stain results for RUNX3 and SMAD4 in PDACs and normal pancreatic parenchyma. (A) RUNX3 is not expressed in normal pancreatic tissue. A few scattered lymphocytes show nuclear RUNX3 expression. Representative PDACs with (B) no nuclear RUNX3 expression and (C) RUNX3 expression. (D) Intact SMAD4 expression in normal pancreatic tissue. PDACs with (E) SMAD4 loss, and (F) intact SMAD4 expression (original magnification x400). 
Table 2: RUNX3 and SMAD4 status and clinicopathological features in pancreatic ductal adenocarcinoma cases

\begin{tabular}{|c|c|c|c|c|c|c|}
\hline Parameters & $\begin{array}{c}\text { SMAD4 } \\
\text { loss } \\
\text { (SMAD4-) }\end{array}$ & $\begin{array}{c}\text { Intact SMAD4 } \\
\text { (SMAD4+) }\end{array}$ & $p$-value & $\begin{array}{l}\text { No RUNX3 } \\
\text { expression } \\
\text { (RUNX3-) }\end{array}$ & $\begin{array}{c}\text { RUNX3 } \\
\text { expression } \\
\text { (RUNX3+) }\end{array}$ & $p$-value \\
\hline Sex & & & 0.747 & & & 0.774 \\
\hline Male & $88(60.7 \%)$ & $32(58.2 \%)$ & & $49(58.3 \%)$ & $73(60.3 \%)$ & \\
\hline Female & $57(39.3 \%)$ & $23(41.8 \%)$ & & $35(41.7 \%)$ & $48(39.7 \%)$ & \\
\hline $\begin{array}{l}\text { Age at operation } \\
(\text { mean } \pm \mathrm{SD})\end{array}$ & $65.1 \pm 9.94$ & $62.3 \pm 9.92$ & 0.082 & $65.3 \pm 8.61$ & $63.3 \pm 10.96$ & 0.146 \\
\hline Tumor size & & & 0.030 & & & 0.039 \\
\hline$\leq 2 \mathrm{~cm}$ & $12(8.3 \%)$ & $12(21.8 \%)$ & & $8(9.5 \%)$ & $17(14.0 \%)$ & \\
\hline$>2 \mathrm{~cm}$ and $\leq 4 \mathrm{~cm}$ & $90(62.0 \%)$ & $28(50.9 \%)$ & & $58(69.0 \%)$ & $62(51.2 \%)$ & \\
\hline$>4 \mathrm{~cm}$ & $43(29.7 \%)$ & $15(27.3 \%)$ & & $18(21.4 \%)$ & $42(34.7 \%)$ & \\
\hline Histologic grade & & & 0.051 & & & 0.033 \\
\hline WD/MD & $122(84.1 \%)$ & $52(94.5 \%)$ & & $78(92.9 \%)$ & $100(82.6 \%)$ & \\
\hline PD/UD & $23(15.9 \%)$ & $3(5.5 \%)$ & & $6(7.1 \%)$ & $21(17.4 \%)$ & \\
\hline Lymphatic invasion & & & 0.665 & & & 0.973 \\
\hline Absent & $81(56.3 \%)$ & $29(52.7 \%)$ & & $46(54.8 \%)$ & $66(55.0 \%)$ & \\
\hline Present & $63(43.7 \%)$ & $26(47.3 \%)$ & & $38(45.2 \%)$ & $54(45.0 \%)$ & \\
\hline Venous invasion & & & 0.591 & & & 0.228 \\
\hline Absent & $91(63.2 \%)$ & $37(67.3 \%)$ & & $58(69.0 \%)$ & $73(60.8 \%)$ & \\
\hline Present & $53(36.8 \%)$ & $18(32.7 \%)$ & & $26(31.0 \%)$ & $47(39.2 \%)$ & \\
\hline Perineural invasion & & & 0.850 & & & 0.926 \\
\hline Absent & $22(15.3 \%)$ & $9(16.4 \%)$ & & $13(15.5 \%)$ & $18(15.0 \%)$ & \\
\hline Present & $122(84.7 \%)$ & $46(83.6 \%)$ & & $71(84.5 \%)$ & $102(85.0 \%)$ & \\
\hline $\begin{array}{l}\text { pT stage } \\
\text { (AJCC 7th Ed.) }\end{array}$ & & & 0.725 & & & 0.509 \\
\hline pT1 & $1(0.7 \%)$ & $0(0.0 \%)$ & & $1(1.2 \%)$ & $0(0.0 \%)$ & \\
\hline pT2 & $4(2.8 \%)$ & $1(1.8 \%)$ & & $3(3.6 \%)$ & $2(1.7 \%)$ & \\
\hline pT3 & $138(95.2 \%)$ & $54(98.2 \%)$ & & $79(94.0 \%)$ & $118(97.5 \%)$ & \\
\hline pT4 & $2(1.4 \%)$ & $0(0.0 \%)$ & & $1(1.2 \%)$ & $1(0.8 \%)$ & \\
\hline $\begin{array}{l}\text { pT stage } \\
\text { (AJCC 8th Ed.) }\end{array}$ & & & 0.054 & & & 0.115 \\
\hline pT1 & $12(8.3 \%)$ & $12(21.8 \%)$ & & $8(9.5 \%)$ & $17(14.0 \%)$ & \\
\hline pT2 & $89(61.4 \%)$ & $28(50.9 \%)$ & & $57(67.9 \%)$ & $62(51.2 \%)$ & \\
\hline pT3 & $42(29.0 \%)$ & $15(27.3 \%)$ & & $18(21.4 \%)$ & $41(33.9 \%)$ & \\
\hline pT4 & $2(1.4 \%)$ & $0(0.0 \%)$ & & $1(1.2 \%)$ & $1(0.8 \%)$ & \\
\hline $\begin{array}{l}\text { pN stage } \\
\text { (AJCC 7th Ed.) }\end{array}$ & & & 0.493 & & & 0.202 \\
\hline pNo & $52(36.6 \%)$ & $23(41.8 \%)$ & & $28(33.3 \%)$ & $51(42.1 \%)$ & \\
\hline $\mathrm{pN} 1$ & $93(63.4 \%)$ & $32(58.2 \%)$ & & $56(66.7 \%)$ & $70(57.9 \%)$ & \\
\hline $\begin{array}{l}\text { pN stage } \\
\text { (AJCC 8th Ed.) }\end{array}$ & & & 0.035 & & & 0.427 \\
\hline pN0 & $53(36.6 \%)$ & $23(41.8 \%)$ & & $28(33.3 \%)$ & $51(42.1 \%)$ & \\
\hline $\mathrm{pN} 1$ & $73(50.3 \%)$ & $18(32.7 \%)$ & & $42(50.0 \%)$ & $51(42.1 \%)$ & \\
\hline pN2 & $19(13.1 \%)$ & $14(25.5 \%)$ & & $14(16.7 \%)$ & $19(15.7 \%)$ & \\
\hline Distant metastasis & & & 0.058 & & & 0.427 \\
\hline Absent & $55(37.9 \%)$ & $29(52.7 \%)$ & & $38(45.2 \%)$ & $48(39.7 \%)$ & \\
\hline Present & $90(62.1 \%)$ & $26(47.3 \%)$ & & $46(54.8 \%)$ & $73(60.3 \%)$ & \\
\hline Local recurrence & & & 0.364 & & & 0.682 \\
\hline Absent & $82(56.6 \%)$ & $35(63.6 \%)$ & & $51(60.7 \%)$ & $70(57.9 \%)$ & \\
\hline Present & $63(43.4 \%)$ & $20(36.4 \%)$ & & $33(39.3 \%)$ & $51(42.1 \%)$ & \\
\hline
\end{tabular}

\section{DISCUSSION}

The purpose of this study was to evaluate the expression status of SMAD4 and RUNX3 in PDACs, and to explore the utility of the SMAD4/RUNX3 marker combination assay in predicting PDAC behavior. We demonstrate in this retrospective study of 210 PDACs that the combination of intact SMAD4 and RUNX3 negativity may help to identify a subset of PDAC patients with favorable prognosis. 
Table 3: Relationship between RUNX3/SMAD4 combined status and clinicopathological features in pancreatic ductal adenocarcinoma cases

\begin{tabular}{|c|c|c|c|}
\hline Parameters & RUNX3-/SMAD4+ & $\begin{array}{c}\text { Others* } \\
\text { (RUNX3+SMAD4+, SMAD4- groups) }\end{array}$ & $p$-value \\
\hline Sex & & & 0.383 \\
\hline Male & $13(52.0 \%)$ & $107(61.1 \%)$ & \\
\hline Female & $12(48.0 \%)$ & $68(38.9 \%)$ & \\
\hline $\begin{array}{l}\text { Age at operation } \\
(\text { mean } \pm \text { SD) }\end{array}$ & $65.2 \pm 8.36$ & $64.2 \pm 10.22$ & 0.605 \\
\hline Tumor size & & & 0.092 \\
\hline$\leq 2 \mathrm{~cm}$ & $5(20.0 \%)$ & $19(10.9 \%)$ & \\
\hline$>2 \mathrm{~cm}$ and $\leq 4 \mathrm{~cm}$ & $17(68.0 \%)$ & $101(57.7 \%)$ & \\
\hline$>4 \mathrm{~cm}$ & $3(12.0 \%)$ & $55(31.4 \%)$ & \\
\hline Histologic grade & & & 0.153 \\
\hline WD/MD & $24(96.0 \%)$ & $150(85.7 \%)$ & \\
\hline $\mathrm{PD} / \mathrm{UD}$ & $1(4.0 \%)$ & $25(14.3 \%)$ & \\
\hline Lymphatic invasion & & & 0.611 \\
\hline Absent & $15(60.0 \%)$ & $95(54.6 \%)$ & \\
\hline Present & $10(40.0 \%)$ & $79(45.4 \%)$ & \\
\hline Venous invasion & & & 0.681 \\
\hline Absent & $17(68.0 \%)$ & $111(63.8 \%)$ & \\
\hline Present & $8(32.0 \%)$ & $63(36.2 \%)$ & \\
\hline Perineural invasion & & & 1.000 \\
\hline Absent & $4(16.0 \%)$ & $27(15.5 \%)$ & \\
\hline Present & $21(84.0 \%)$ & $147(84.5 \%)$ & \\
\hline pT stage (AJCC 7th Ed.) & & & 0.755 \\
\hline pT1 & $0(0.0 \%)$ & $1(0.6 \%)$ & \\
\hline pT2 & $0(0.0 \%)$ & $5(2.9 \%)$ & \\
\hline pT3 & $25(100.0 \%)$ & $167(95.4 \%)$ & \\
\hline pT4 & $0(0.0 \%)$ & $2(1.1 \%)$ & \\
\hline pT stage (AJCC 8th Ed.) & & & 0.173 \\
\hline pT1 & $5(20.0 \%)$ & $19(10.9 \%)$ & \\
\hline pT2 & $17(68.0 \%)$ & $100(57.1 \%)$ & \\
\hline pT3 & $3(12.0 \%)$ & $54(30.9 \%)$ & \\
\hline pT4 & $0(0.0 \%)$ & $2(1.1 \%)$ & \\
\hline pN stage (AJCC 7th Ed.) & & & 0.509 \\
\hline $\mathrm{pNO}$ & $11(44.0 \%)$ & $65(37.1 \%)$ & \\
\hline pN1 & $14(56.0 \%)$ & $110(62.9 \%)$ & \\
\hline pN stage (AJCC 8th Ed.) & & & 0.592 \\
\hline pNO & $11(44.0 \%)$ & $65(37.1 \%)$ & \\
\hline $\mathrm{pN} 1$ & $9(36.0 \%)$ & $82(46.9 \%)$ & \\
\hline $\mathrm{pN} 2$ & $5(20.0 \%)$ & $28(16.0 \%)$ & \\
\hline Distant metastasis & & & 0.017 \\
\hline Absent & $16(64.0 \%)$ & $68(38.9 \%)$ & \\
\hline Present & $9(36.0 \%)$ & $107(61.1 \%)$ & \\
\hline Local recurrence & & & 0.303 \\
\hline Absent & $17(68.0 \%)$ & $100(57.1 \%)$ & \\
\hline Present & $8(32.0 \%)$ & $75(42.9 \%)$ & \\
\hline
\end{tabular}

*Others: RUNX3-/SMAD4-, RUNX3+/SMAD4-, RUNX3+/SMAD4+.

SMAD4 (or DPC4) is a well-known tumor suppressor gene that is inactivated in the late stages of pancreatic carcinogenesis: SMAD4 inactivation has been reported in $\sim 50 \%$ of PDACs and in $~ 30 \%$ of highgrade pancreatic intraepithelial neoplasia (PanIN-3)
[9-12]. Although the majority of clinicopathological studies, including our present study, have demonstrated associations between SMAD4 loss in PDACs and poor prognoses, there are also reports showing no significant correlations with survival, or even evidence for improved 
Table 4: Survival analysis in clinicopathological variables and immunohistochemical results

\begin{tabular}{|c|c|c|c|c|c|c|}
\hline \multirow[b]{2}{*}{ Parameters } & \multicolumn{3}{|c|}{ Overall survival } & \multicolumn{3}{|c|}{ Progression-free survival } \\
\hline & $\begin{array}{l}\text { Median survival time } \\
\text { (month, } \\
95 \% \mathrm{CI} \text { ) }\end{array}$ & Number of events & $p$-value & $\begin{array}{l}\text { Median survival time } \\
\text { (month, } \\
95 \% \mathrm{CI} \text { ) }\end{array}$ & Number of events & $p$-value \\
\hline Sex & & & 0.169 & & & 0.189 \\
\hline Male & $\begin{array}{c}19.00 \\
(15.81-22.18)\end{array}$ & $111 / 126$ & & $\begin{array}{c}10.00 \\
(6.10-13.89)\end{array}$ & $113 / 125$ & \\
\hline Female & $\begin{array}{c}17.00 \\
(11.40-22.59)\end{array}$ & $63 / 84$ & & $\begin{array}{c}8.00 \\
(6.36-9.63)\end{array}$ & $67 / 84$ & \\
\hline Age at operation & & & 0.376 & & & 0.715 \\
\hline$\leq 65$ years & $\begin{array}{c}19.00 \\
(13.96-24.03)\end{array}$ & $88 / 108$ & & $\begin{array}{c}10.00 \\
(6.40-13.59)\end{array}$ & $91 / 107$ & \\
\hline$<65$ years & $\begin{array}{c}16.00 \\
(12.27-19.72)\end{array}$ & $86 / 102$ & & $\begin{array}{c}8.00 \\
(5.67-10.32)\end{array}$ & $89 / 102$ & \\
\hline Tumor size & & & 0.032 & & & 0.013 \\
\hline$\leq 2 \mathrm{~cm}$ & $\begin{array}{c}29.00 \\
(19.00-38.99)\end{array}$ & $20 / 26$ & & $\begin{array}{c}16.00 \\
(9.82-22.17)\end{array}$ & $21 / 26$ & \\
\hline$>2 \mathrm{~cm}$ and $\leq 4 \mathrm{~cm}$ & $\begin{array}{c}19.00 \\
(15.40-22.59)\end{array}$ & $103 / 123$ & & $\begin{array}{c}10.00 \\
(5.67-14.32)\end{array}$ & $105 / 122$ & \\
\hline$>4 \mathrm{~cm}$ & $\begin{array}{c}12.00 \\
(8.79-15.20)\end{array}$ & $51 / 61$ & & $\begin{array}{c}5.00 \\
(3.49-6.50)\end{array}$ & $54 / 61$ & \\
\hline Histologic grade & & & 0.124 & & & 0.391 \\
\hline $\mathrm{WD} / \mathrm{MD}$ & $\begin{array}{c}19.00 \\
(15.59-22.40)\end{array}$ & $150 / 182$ & & $\begin{array}{c}9.00 \\
(6.89-11.10)\end{array}$ & $157 / 182$ & \\
\hline $\mathrm{PD} / \mathrm{UD}$ & $\begin{array}{c}11.00 \\
(7.12-14.87)\end{array}$ & $24 / 28$ & & $\begin{array}{c}5.00 \\
(1.60-8.39)\end{array}$ & $23 / 27$ & \\
\hline Lymphatic invasion & & & 0.005 & & & 0.003 \\
\hline Absent & $\begin{array}{c}21.00 \\
(16.51-25.48)\end{array}$ & $91 / 114$ & & $\begin{array}{c}15.00 \\
(10.83-19.16)\end{array}$ & $94 / 113$ & \\
\hline Present & $\begin{array}{c}14.00 \\
(11.44-16.44)\end{array}$ & $83 / 95$ & & $\begin{array}{c}6.00 \\
(3.96-8.03)\end{array}$ & $86 / 95$ & \\
\hline Venous invasion & & & $<0.001$ & & & 0.003 \\
\hline Absent & $\begin{array}{c}23.00 \\
(19.05-26.94)\end{array}$ & $106 / 134$ & & $\begin{array}{c}11.00 \\
(6.83-15.16)\end{array}$ & $111 / 133$ & \\
\hline Present & $\begin{array}{c}13.00 \\
(10.52-15.47)\end{array}$ & $68 / 75$ & & $\begin{array}{c}6.00 \\
(4.14-7.86)\end{array}$ & $69 / 75$ & \\
\hline Perineural invasion & & & 0.007 & & & 0.007 \\
\hline Absent & $\begin{array}{c}24.00 \\
(0.00-59.25)\end{array}$ & $20 / 32$ & & $\begin{array}{c}19.00 \\
(2.36-35.63)\end{array}$ & $22 / 32$ & \\
\hline Present & $\begin{array}{c}17.00 \\
(14.66-19.34)\end{array}$ & $154 / 177$ & & $\begin{array}{c}8.00 \\
(6.00-9.99)\end{array}$ & $158 / 176$ & \\
\hline pT stage (7th Ed.) & & & 0.041 & & & 0.014 \\
\hline pT1 & 8.00 & $1 / 2$ & & 8.00 & $1 / 2$ & \\
\hline pT2 & $*$ & $1 / 5$ & & $*$ & $1 / 5$ & \\
\hline pT3 & $\begin{array}{c}18.00 \\
(15.62-20.37)\end{array}$ & $170 / 201$ & & $\begin{array}{c}9.00 \\
(7.09-10.90)\end{array}$ & $176 / 200$ & \\
\hline pT4 & 14.00 & $2 / 2$ & & $*$ & $2 / 2$ & \\
\hline pT stage (8th Ed.) & & & 0.055 & & & 0.018 \\
\hline pT1 & $\begin{array}{c}29.00 \\
(19.00-38.99)\end{array}$ & $20 / 26$ & & $\begin{array}{c}16.00 \\
(9.82-22.17)\end{array}$ & $21 / 26$ & \\
\hline pT2 & $\begin{array}{c}19.00 \\
(15.42-22.57)\end{array}$ & $102 / 122$ & & $\begin{array}{c}10.00 \\
(5.69-14.30)\end{array}$ & $104 / 121$ & \\
\hline pT3 & $\begin{array}{c}12.00 \\
(8.82-15.17)\end{array}$ & $50 / 60$ & & $\begin{array}{c}5.00 \\
(3.39-6.60)\end{array}$ & $53 / 60$ & \\
\hline pT4 & 14.00 & $2 / 2$ & & 4.00 & $2 / 2$ & \\
\hline pN stage (7th Ed.) & & & $<0.001$ & & & 0.003 \\
\hline $\mathrm{pN} 0$ & $\begin{array}{c}27.00 \\
(16.02-37.97)\end{array}$ & $59 / 80$ & & $\begin{array}{c}14.00 \\
(7.22-20.77)\end{array}$ & $63 / 79$ & \\
\hline $\mathrm{pN} 1$ & $\begin{array}{c}16.00 \\
(13.64-18.35)\end{array}$ & $115 / 130$ & & $\begin{array}{c}8.00 \\
(6.04-9.95)\end{array}$ & $117 / 130$ & \\
\hline
\end{tabular}


pN stage (8th Ed.)

$\mathrm{pN} 0$
$\mathrm{pN} 1$
$\mathrm{pN} 2$

SMAD4 status

Loss

Intact

RUNX3 status

No expression

Expression

4-group combination

RUNX3-/SMAD4-

RUNX3-/SMAD4+

RUNX3+/SMAD4-

RUNX3+/SMAD4+

2-group combination

RUNX3-/SMAD4+

Others**
27.00

(16.02-37.97)

15.00

(12.62-17.37)

17.00

(13.78-20.21)

$$
\begin{gathered}
16.00 \\
(13.54-18.45) \\
25.00
\end{gathered}
$$

(19.15-30.84)

23.00

(18.51-27.48)

16.00

(13.57-18.42)

19.00

(14.92-23.07)

34.00

(12.78-55.21)

15.00

(12.74-17.25)

20.00

(13.72-26.27)

34.00

(12.78-55.21)

17.00

(14.72-19.27)
$59 / 80$

$85 / 97$

$30 / 33$

$127 / 145$

$39 / 55$

$66 / 84$

$104 / 121$

$48 / 56$

$16 / 25$

79/89

$23 / 30$

$16 / 25$

$150 / 175$
0.001

0.011

14.00
$(7.22-20.77)$
8.00
$(6.41-9.58)$
9.00
$(4.98-13.01)$

$63 / 79$

$87 / 97$

$30 / 33$

0.015

8.00

(5.95-10.04)

11.00

(6.21-15.78)

0.010

12.00

(5.41-18.58)

8.00

(5.53-10.46)

0.015

8.00

(0.67-15.32)

29.00

(0.00-58.37)

8.00

(5.70-10.29)

7.00

(1.72-12.27)

0.008

29.00

(0.00-58.37)

8.00

(6.01-9.98)
0.044

$130 / 144$

$42 / 55$

0.009

$68 / 84$

$108 / 120$

0.030

49/56

$17 / 25$

$81 / 88$

$25 / 30$

0.009

* All cases were censored.

** RUNX3-/SMAD4-, RUNX3+/SMAD4-, RUNX3+/SMAD4+.

survival in PDACs with SMAD4 loss [13-17]. Loss of SMAD4 protein expression has been demonstrated to be a sensitive and specific surrogate marker for SMAD4 alteration, independent of whether SMAD4 inactivation occurred by homozygous deletions or by loss of heterozygosity, and the presence of intact SMAD4 protein expression corresponds to tumors with at least one wildtype allele (i.e. SMAD4(+/+) or SMAD4(+/-)) $[11,18]$. Therefore, PDACs with intact SMAD4 protein expression would comprise a mixed population of $S M A D 4(+/+)$ and SMAD4(+/-) PDACs, and hence the discrepancies in the clinicopathological studies on the prognostic significance of SMAD4 immunohistochemistry are not surprising. In addition, PDACs with SMAD4 loss tended to show more frequent distant metastasis on follow-up compared to SMAD4-intact tumors, while there was no difference in the frequency of local progression according to SMAD4 status, supporting the previous literature that suggested correlations between SMAD4 status and disease progression patterns in PDAC [19, 20].

The role of RUNX3 in cancer is still not wellestablished; it has been described to function as a tumor suppressor in most malignancies, including cancers of the breast, stomach, liver, lung and prostate, [21-25] but tumor promoting roles of RUNX3 have been suggested for some tumors such as ovarian cancers, melanomas and PDACs [8, 26-28]. An association between RUNX3 protein expression and decreased overall survival has also been demonstrated in PDAC patients, although in a small number of cases [8]. A recent genetically engineered mouse model study by Whittle et al. demonstrated an interesting relationship between SMAD4 and RUNX3 status and the biological behavior (metastasis versus primary tumor growth) of PDACs according to SMAD4/ RUNX3 status [7, 8, 29]. In brief, KPC (SMAD4(+/+)) and KPDDC (SMAD4(-/-)) tumors showed increased $R U N X 3$ expression levels and a high metastatic disease burden, and the high expression of RUNX3 was associated with increased metastatic potential of PDACs independent of epithelial-mesenchymal transition [8, 30]. On the other hand, SMAD4 haploinsufficient KPDC mice $(S M A D 4(+/-))$ lacked RUNX3 expression, had a significantly lower metastatic burden, but showed an increased proliferative activity of the primary tumor compared to KPC tumors. We did not find differences in the proliferative activity (Ki-67 labeling index) of PDACs according to SMAD4/RUNX3 status in this study (data not shown), but we did find that PDACs with RUNX3 
expression and/or SMAD4 loss were more likely to metastasize to distant sites and follow an aggressive clinical course compared to PDACs that did not show these immunophenotypes. Therefore, combining the SMAD4 and RUNX3 protein expression status would provide a more accurate prognostic marker compared to SMAD4 status alone.

It is also interesting that when the immunohistochemical profiles of the primary and metastatic tumors were compared in the separate analysis of 14 primary versus matched metastatic PDACs, SMAD4 labeling status was concordant in the majority of the cases, while the RUNX3 expression status were more frequently discordant. All primary tumors showed
RUNX3 expression and/or SMAD4 loss; RUNX3-/SMAD4+ status was not seen in any of the primary or metastatic tumors in this analysis. In addition, all RUNX3-discordant cases showed conversion from RUNX3-positivity in the primary tumor to RUNX3-negativity in the matched metastatic tumors. Attenuation of RUNX3 levels once the metastatic niche is established, switching the metastatic tumor to the locally proliferative state, would be an attractive explanation for this observation; however, experimental evidence would be required to support this.

This is to our knowledge the first and the largest single institutional clinicopathological study demonstrating RUNX3/SMAD4 combination


Figure 2: Survival analysis results. Decreased overall survival (OS) (A) and progression-free survival (PFS) (B) are seen in PDACs with SMAD4 loss compared to those with intact SMAD4. RUNX3-expressing PDACs demonstrate decreased OS (C) and PFS (D) compared to RUNX3-negative tumors. RUNX3-/SMAD4+ PDACs show increased OS (E, G) and PFS (F, H) compared to the other PDACs. (G, H) "Other groups": RUNX3-/SMAD4-, RUNX3+/SMAD4-, RUNX3+/SMAD4+ PDACs). 
Table 5: Multivariate analysis for overall survival and progression-free survival

\begin{tabular}{|c|c|c|c|c|}
\hline \multirow[t]{2}{*}{ Parameters } & \multicolumn{2}{|c|}{ Overall survival } & \multicolumn{2}{|c|}{ Progression-free survival } \\
\hline & $\begin{array}{c}\text { Hazard ratio } \\
(95 \% \mathrm{CI})\end{array}$ & $p$-value & $\begin{array}{c}\text { Hazard ratio } \\
(95 \% \mathrm{CI})\end{array}$ & $p$-value \\
\hline \multicolumn{5}{|c|}{ Variables adjusted for age and sex } \\
\hline $\begin{array}{l}\text { RUNX3-/SMAD4+ vs. } \\
\text { others* }\end{array}$ & $1.842(1.079-3.143)$ & 0.025 & $1.850(1.100-3.113)$ & 0.020 \\
\hline Histologic grade & $1.517(0.961-2.393)$ & 0.073 & $1.163(0.730-1.852)$ & 0.525 \\
\hline \multicolumn{5}{|l|}{ Tumor size } \\
\hline$\leq 2 \mathrm{~cm}$ & 1 & Reference & 1 & Reference \\
\hline$>2 \mathrm{~cm}$ and $\leq 4 \mathrm{~cm}$ & $1.511(0.912-2.503)$ & 0.109 & $1.298(0.787-2.142)$ & 0.307 \\
\hline$>4 \mathrm{~cm}$ & $2.251(1.287-3.935)$ & 0.004 & $2.191(1.248-3.847)$ & 0.006 \\
\hline Lymphatic invasion & $1.330(0.962-1.839)$ & 0.085 & $1.492(1.101-2.023)$ & 0.010 \\
\hline Venous invasion & $1.547(1.100-2.176)$ & 0.012 & $1.267(0.896-1.792)$ & 0.180 \\
\hline Perineural invasion & $1.546(0.935-2.557)$ & 0.089 & $1.565(0.982-2.494)$ & 0.059 \\
\hline pT stage (7th Ed.) & $8.008(1.071-59.866)$ & 0.043 & $10.211(1.380-75.567)$ & 0.023 \\
\hline pN stage (7th Ed.) & $1.178(0.837-1.658)$ & 0.348 & $1.057(0.758-1.474)$ & 0.745 \\
\hline
\end{tabular}

* RUNX3-/SMAD4-, RUNX3+/SMAD4-, RUNX3+/SMAD4+.

immunohistochemistry to be a useful predictor of both overall and disease-free survivals in PDAC patients. Although further validation in independent cohorts would be required, application of RUNX3/ SMAD4 immunohistochemistry on biopsies or resected PDAC tissues may not only be a prognostic marker but also help guide treatment plans in PDAC patients; RUNX3-/SMAD4+ PDACs are less likely to metastasize to distant sites and may therefore be candidates for more aggressive local surgery and radiotherapy, whereas PDACs with RUNX3 expression and/or SMAD4 loss profiles may benefit more from systemic chemotherapy.

\section{MATERIALS AND METHODS}

\section{Patient selection and clinicopathological analysis}

This retrospective analysis was approved by the Institutional Review Board of Seoul National University Bundang Hospital (IRB \#B-1611-369-302). The study subjects comprised 210 consecutive cases of PDACs resected between June 2003 and January 2013 at Seoul National University Bundang Hospital, Seongnam, South Korea. Clinicopathological data were retrieved by reviewing electronic medical records, pathology reports and glass slides, and included patient sex, initial treatment, age at operation, tumor size, histologic differentiation, serum tumor marker levels (CEA, CA19-9), and pathological $\mathrm{T}$ and $\mathrm{N}$ stages according to American Joint Committee on Cancer (AJCC) 7th edition and the new 8th edition. Follow up data was also retrieved from the electronic medical records, including the status at last follow up, and occurrence of local recurrence and/or distant metastasis during the follow up period. The dates of local recurrence and distant metastasis were defined as when new lesions appeared on imaging studies or when tumor marker levels were elevated. The date of disease progression was defined as when the event has occurred postoperatively such as local recurrence, distant metastasis or death.

\section{Immunohistochemistry}

Two mm-core tissue microarrays were constructed from 210 PDACs and their matched non-neoplastic pancreatic tissues (Superbiochips Laboratories, Seoul, Korea), and $4 \mu \mathrm{m}$-thick tissue sections obtained from the microarray blocks were subjected to immunohistochemical staining for SMAD4 (1:100, rabbit monoclonal, Abcam, Cambridge, UK) and RUNX3 (1:100, rabbit monoclonal, Cell signaling technology, Denver, USA). In brief, tissue sections were deparaffinized in xylene, rehydrated in graded alcohol, and antigen retrieval was performed using citrate buffer ( $\mathrm{pH}$ 6.0). Incubation with primary antibodies was performed for 1 hour, and then with secondary antibodies (EnVision Detection System, Dako) for 30 minutes. Counterstaining was performed using Mayer's hematoxylin.

The labeled slides were interpreted by 2 pathologists (YL and HK). SMAD4 was expressed in the cytoplasm and nuclei of normal pancreatic duct epithelia, acinar cells, lymphocytes, stromal fibroblasts and endocrine cells, and served as positive controls. Expression in $<20 \%$ of tumor cell nuclei was defined as SMAD4 loss ("SMAD4-"). RUNX3 was not expressed in normal pancreatic ductal epithelial cells or acinar cells, but was expressed in the 
Table 6: Comparison of SMAD4 and RUNX3 labeling status between primary and metastatic tumors $(n=14)$

\begin{tabular}{cccc}
\hline Case\# & Status of primary site & Metastatic sites & Status of metastatic site \\
\hline 1 & RUNX3+/SMAD4- & Liver & RUNX3-/SMAD4- \\
2 & RUNX3+/SMAD4- & Liver & RUNX3-/SMAD4- \\
$\mathbf{3}$ & RUNX3+/SMAD4- & Liver & RUNX3+/SMAD4- \\
4 & RUNX3+/SMAD4- & Cervical lymph node & RUNX3-/SMAD4- \\
5 & RUNX3+/SMAD4- & Lung & RUNX3-/SMAD4- \\
6 & RUNX3+/SMAD4- & Liver & RUNX3+/SMAD4+ \\
7 & RUNX3+/SMAD4- & Liver & RUNX3-/SMAD4- \\
$\mathbf{8}$ & RUNX3-/SMAD4- & Liver & RUNX3-/SMAD4- \\
9 & RUNX3+/SMAD4+ & Liver & RUNX3-/SMAD4- \\
$\mathbf{1 0}$ & RUNX3-/SMAD4- & Liver & RUNX3-/SMAD4- \\
$\mathbf{1 1}$ & RUNX3-/SMAD4- & Liver & RUNX3-/SMAD4- \\
12 & RUNX3+/SMAD4+ & Bone & RUNX3-/SMAD4- \\
13 & RUNX3+/SMAD4- & Lung & RUNX3-/SMAD4- \\
$\mathbf{1 4}$ & RUNX3-/SMAD4- & Colon & RUNX3-/SMAD4- \\
\hline
\end{tabular}

lymphocytes. RUNX3 expression status was evaluated in terms of both intensity and distribution of staining. The staining intensity was defined as follows: "0", no expression; "1", weaker intensity compared to staining of lymphocytes; " 2 ", strong intensity similar to that seen in lymphocytes. For distribution, the percentage of positively stained tumor cell nuclei was estimated in 5\% increments. The staining distribution was relatively homogeneous throughout the tumor. The presence of weak or strong RUNX3 positivity in $5 \%$ or more of tumor cells were regarded as positive ("RUNX3+") for subsequent analysis.

For cases where both primary pancreatic tumors and metastatic tumors were available for examination, whole tissue sections were subjected to the same immunohistochemical stains for RUNX3 and SMAD4, and the expression status of both markers were compared between the primary and matched metastatic tumors. These cases were from the same cohort of 210 PDACs.

\section{Statistical analysis}

All statistical analyses were performed using SPSS 19.0K (SPSS Korea, Seoul, South Korea). Chi-square tests, Fisher exact tests and Student $t$-tests were performed as deemed appropriate. Univariate survival analyses for overall and progression-free survivals were performed by the Kaplan-Meier method and log-rank test. The Cox regression models were used for multivariate analysis. Statistical significance was defined as $p<0.05$.

\section{CONFLICTS OF INTEREST}

The authors declare no potential conflicts of interest.

\section{FUNDING}

This study was supported by grant number 02-2016008 from the SNUBH Research Fund, and the Basic Science Research Program through NRF funded by the Ministry of Education (NRF-2016R1D1A1A09919042) to HK.

\section{REFERENCES}

1. Siegel RL, Miller KD, Jemal A. Cancer statistics, 2016. CA Cancer J Clin. 2016; 66:7-30. https://doi.org/10.3322/caac.21332.

2. Jones S, Zhang X, Parsons DW, Lin JC, Leary RJ, Angenendt P, Mankoo P, Carter H, Kamiyama H, Jimeno A, Hong SM, Fu B, Lin MT, et al. Core signaling pathways in human pancreatic cancers revealed by global genomic analyses. Science. 2008; 321:1801-6. https://doi. org/10.1126/science.1164368.

3. Seymour AB, Hruban RH, Redston M, Caldas C, Powell SM, Kinzler KW, Yeo CJ, Kern SE. Allelotype of pancreatic adenocarcinoma. Cancer Res. 1994; 54:2761-4.

4. Bailey P, Chang DK, Nones K, Johns AL, Patch AM, Gingras MC, Miller DK, Christ AN, Bruxner TJ, Quinn MC, Nourse C, Murtaugh LC, Harliwong I, et al. Genomic analyses identify molecular subtypes of pancreatic cancer. Nature. 2016; 531:47-52. https://doi.org/10.1038/ nature 16965.

5. Collisson EA, Sadanandam A, Olson P, Gibb WJ, Truitt M, Gu S, Cooc J, Weinkle J, Kim GE, Jakkula L, Feiler HS, Ko AH, Olshen AB, et al. Subtypes of pancreatic ductal adenocarcinoma and their differing responses to therapy. Nat Med. 2011; 17:500-3. https://doi.org/10.1038/ nm.2344. 
6. Moffitt RA, Marayati R, Flate EL, Volmar KE, Loeza SG, Hoadley KA, Rashid NU, Williams LA, Eaton SC, Chung AH, Smyla JK, Anderson JM, Kim HJ, et al. Virtual microdissection identifies distinct tumor- and stroma-specific subtypes of pancreatic ductal adenocarcinoma. Nat Genet. 2015; 47:116878. https://doi.org/10.1038/ng.3398.

7. Whittle MC, Hingorani SR. Runx 3 and Cell Fate Decisions in Pancreas Cancer. Adv Exp Med Biol. 2017; 962:333-52. https://doi.org/10.1007/978-981-10-3233-2_21.

8. Whittle MC, Izeradjene K, Rani PG, Feng L, Carlson MA, DelGiorno KE, Wood LD, Goggins M, Hruban RH, Chang AE, Calses P, Thorsen SM, Hingorani SR. RUNX3 Controls a Metastatic Switch in Pancreatic Ductal Adenocarcinoma. Cell. 2015; 161:1345-60. https://doi. org/10.1016/j.cell.2015.04.048.

9. Demagny H, De Robertis EM. Point mutations in the tumor suppressor Smad4/DPC4 enhance its phosphorylation by GSK3 and reversibly inactivate TGF-beta signaling. Mol Cell Oncol. 2016; 3:e1025181. https://doi.org/10.1080/237 23556.2015.1025181.

10. Hahn SA, Schutte M, Hoque AT, Moskaluk CA, da Costa LT, Rozenblum E, Weinstein CL, Fischer A, Yeo CJ, Hruban RH, Kern SE. DPC4, a candidate tumor suppressor gene at human chromosome 18q21.1. Science. 1996; 271:350-3.

11. Schutte M, Hruban RH, Hedrick L, Cho KR, Nadasdy GM, Weinstein CL, Bova GS, Isaacs WB, Cairns P, Nawroz H, Sidransky D, Casero RA Jr, Meltzer PS, et al. DPC4 gene in various tumor types. Cancer Res. 1996; 56:2527-30.

12. Wilentz RE, Iacobuzio-Donahue CA, Argani $P$, McCarthy DM, Parsons JL, Yeo CJ, Kern SE, Hruban RH. Loss of expression of Dpc4 in pancreatic intraepithelial neoplasia: evidence that DPC4 inactivation occurs late in neoplastic progression. Cancer Res. 2000; 60:2002-6.

13. Biankin AV, Morey AL, Lee CS, Kench JG, Biankin SA, Hook HC, Head DR, Hugh TB, Sutherland RL, Henshall SM. DPC4/Smad4 expression and outcome in pancreatic ductal adenocarcinoma. J Clin Oncol. 2002; 20:4531-42. https://doi.org/10.1200/JCO.2002.12.063.

14. Bachet JB, Marechal R, Demetter P, Bonnetain F, Couvelard A, Svrcek M, Bardier-Dupas A, Hammel P, Sauvanet A, Louvet C, Paye F, Rougier P, Penna C, et al. Contribution of CXCR4 and SMAD4 in predicting disease progression pattern and benefit from adjuvant chemotherapy in resected pancreatic adenocarcinoma. Ann Oncol. 2012; 23:2327-35. https://doi.org/10.1093/annonc/mdr617.

15. Tascilar M, Skinner HG, Rosty C, Sohn T, Wilentz RE, Offerhaus GJ, Adsay V, Abrams RA, Cameron JL, Kern SE, Yeo CJ, Hruban RH, Goggins M. The SMAD4 protein and prognosis of pancreatic ductal adenocarcinoma. Clin Cancer Res. 2001; 7:4115-21.

16. Shugang $\mathrm{X}$, Hongfa $\mathrm{Y}$, Jianpeng $\mathrm{L}, \mathrm{Xu} \mathrm{Z}$, Jingqi $\mathrm{F}$, Xiangxiang L, Wei L. Prognostic Value of SMAD4 in Pancreatic Cancer: A Meta-Analysis. Transl Oncol. 2016; 9:1-7. https://doi.org/10.1016/j.tranon.2015.11.007.
17. Wang JD, Jin $\mathrm{K}$, Chen XY, Lv JQ, Ji KW. Clinicopathological significance of SMAD4 loss in pancreatic ductal adenocarcinomas: a systematic review and meta-analysis. Oncotarget. 2017; 8:16704-11. https:// doi.org/10.18632/oncotarget.14335.

18. Wilentz RE, Su GH, Dai JL, Sparks AB, Argani P, Sohn TA, Yeo CJ, Kern SE, Hruban RH. Immunohistochemical labeling for dpc4 mirrors genetic status in pancreatic adenocarcinomas : a new marker of DPC4 inactivation. Am J Pathol. 2000; 156:37-43. https://doi.org/10.1016/S00029440(10)64703-7.

19. Crane CH, Varadhachary GR, Yordy JS, Staerkel GA, Javle MM, Safran H, Haque W, Hobbs BD, Krishnan S, Fleming JB, Das P, Lee JE, Abbruzzese JL, et al. Phase II trial of cetuximab, gemcitabine, and oxaliplatin followed by chemoradiation with cetuximab for locally advanced (T4) pancreatic adenocarcinoma: correlation of Smad4(Dpc4) immunostaining with pattern of disease progression. J Clin Oncol. 2011; 29:3037-43. https://doi.org/10.1200/ JCO.2010.33.8038.

20. Iacobuzio-Donahue $\mathrm{CA}, \mathrm{Fu} \mathrm{B}$, Yachida $\mathrm{S}$, Luo M, Abe $\mathrm{H}$, Henderson CM, Vilardell F, Wang Z, Keller JW, Banerjee P, Herman JM, Cameron JL, Yeo CJ, et al. DPC4 gene status of the primary carcinoma correlates with patterns of failure in patients with pancreatic cancer. J Clin Oncol. 2009; 27:1806-13. https://doi.org/10.1200/ JCO.2008.17.7188

21. Chen F, Wang M, Bai J, Liu Q, Xi Y, Li W, Zheng J. Role of RUNX3 in suppressing metastasis and angiogenesis of human prostate cancer. PLoS One. 2014; 9:e86917. https:// doi.org/10.1371/journal.pone.0086917.

22. Guo WH, Weng LQ, Ito $\mathrm{K}$, Chen LF, Nakanishi $\mathrm{H}$, Tatematsu M, Ito Y. Inhibition of growth of mouse gastric cancer cells by Runx3, a novel tumor suppressor. Oncogene. 2002; 21:8351-5. https://doi.org/10.1038/sj.onc.1206037.

23. Huang B, Qu Z, Ong CW, Tsang YH, Xiao G, Shapiro D, Salto-Tellez M, Ito K, Ito Y, Chen LF. RUNX3 acts as a tumor suppressor in breast cancer by targeting estrogen receptor alpha. Oncogene. 2012; 31:527-34. https://doi. org/10.1038/onc.2011.252.

24. Lee KS, Lee YS, Lee JM, Ito K, Cinghu S, Kim JH, Jang JW, Li YH, Goh YM, Chi XZ, Wee H, Lee HW, Hosoya A, et al. Runx 3 is required for the differentiation of lung epithelial cells and suppression of lung cancer. Oncogene. 2010; 29:3349-61. https://doi.org/10.1038/onc.2010.79.

25. Mori T, Nomoto S, Koshikawa K, Fujii T, Sakai M, Nishikawa Y, Inoue S, Takeda S, Kaneko T, Nakao A. Decreased expression and frequent allelic inactivation of the RUNX3 gene at 1p36 in human hepatocellular carcinoma. Liver Int. 2005; 25:380-8. https://doi.org/10.1111/j.14783231.2005.1059.x.

26. Lee CW, Chuang LS, Kimura S, Lai SK, Ong CW, Yan B, Salto-Tellez M, Choolani M, Ito Y. RUNX3 functions as an oncogene in ovarian cancer. Gynecol Oncol. 2011; 122:410-7. https://doi.org/10.1016/j.ygyno.2011.04.044. 
27. Li J, Kleeff J, Guweidhi A, Esposito I, Berberat PO, Giese T, Buchler MW, Friess H. RUNX3 expression in primary and metastatic pancreatic cancer. J Clin Pathol. 2004; 57:294-9.

28. Nevadunsky NS, Barbieri JS, Kwong J, Merritt MA, Welch WR, Berkowitz RS, Mok SC. RUNX3 protein is overexpressed in human epithelial ovarian cancer. Gynecol Oncol. 2009; 112:325-30. https://doi.org/10.1016/j. ygyno.2008.09.006.
29. Whittle MC, Hingorani SR. RUNX3 defines disease behavior in pancreatic ductal adenocarcinoma. Mol Cell Oncol. 2016; 3:e1076588. https://doi.org/10.1080/237235 56.2015.1076588.

30. Whittle MC, Hingorani SR. Disconnect between EMT and metastasis in pancreas cancer. Oncotarget. 2015; 6:30445-6. https://doi.org/10.18632/oncotarget.5720. 